\title{
Energy Efficient Network Coding-based MAC for Cooperative ARQ Wireless Networks
}

\author{
Angelos Antonopoulos ${ }^{\mathrm{a}, *}$, Christos Verikoukis ${ }^{\mathrm{a}}$, Charalabos Skianis ${ }^{\mathrm{b}}$, Ozgur \\ B. Akan ${ }^{\mathrm{c}}$ \\ ${ }^{a}$ Telecommunications Technological Centre of Catalonia (CTTC), Castelldefels, \\ Barcelona, Spain \\ ${ }^{b}$ Department of Information and Communication Systems Engineering, University of the \\ Aegean, Karlovassi, Greece \\ ${ }^{c}$ Department of Electrical and Electronics Engineering, Koc University, Istanbul, Turkey
}

\section{Abstract}

In this paper we introduce a network coding-aided energy efficient Medium Access Control (MAC) protocol that coordinates the transmissions among a set of relay nodes which act as helpers in cooperative Automatic Repeat reQuest-based (ARQ-based) wireless networks. Applying network coding techniques, we achieve to increase the energy efficiency of the network without compromising the system performance in terms of Quality of Service. Our proposed solution is evaluated by both analytical and simulation results.

Keywords: Network Coding, "Green" Communication, Cooperation, Automatic Repeat reQuest

*Corresponding author. Tel: +34 936452900

E-mail Addresses : aantonopoulos@cttc.es (A. Antonopoulos), cveri@cttc.es (C. Verikoukis), cskianis@aegean.gr (C. Skianis), akan@ku.edu.tr (O. B. Akan) 


\section{Introduction}

Energy efficiency has always been a critical design parameter for wireless networks. Recently, the trend towards designing energy-aware communication protocols has become more intense due to the scarcity of the energy resources. The purpose of these new protocols is twofold: i) to extend the battery's life in portable devices and ii) to efficiently use the environment's natural resources. Therefore, "green" ${ }^{1}$ communications have become one of the hottest topics in the research community.

Cooperation among nodes and network coding are two techniques that have been introduced to improve the network performance and provide the communication with diversity, robustness, security and high data rates. The benefits of these technologies can be exploited by designing new MAC protocols that efficiently use and manage the network resources.

The concept of cooperation was introduced by Cover et al. [1] in their fundamental paper on relay channels. Their work analyzed the capacity of the three-node network consisting of a transmitter, a receiver and a partner ${ }^{2}$. In their model, the spatial diversity gain is obtained by exploiting different channels seen by different nodes for transmitting data.

On the other hand, network coding is an area emerged in 2000 [2]-[3], and since then has attracted an increasing interest, as it promises to have a significant impact in both theory and practice of networks. Network coding can be broadly defined as and advanced routing or encoding mechanism at

\footnotetext{
1 "Green" refers to all environment-aware methods.

${ }^{2}$ Note that the words "partner", "relay" and "helper" are used interchangeably in this paper.
} 
network layer, which allows intermediate nodes in the network not only to forward but also to process the incoming information flows. Most of the work on this topic focuses on the physical layer aspect [4]-[7] while only few works examine these techniques considering the MAC layer effect [8]-[10]. Furthermore, the main assumption in the majority of the works is that only one relay contributes to the communication, thus ignoring the impact of collisions and idle slots to the network performance.

In this context we propose a novel Network Coding-based Cooperative ARQ MAC (NCCARQ-MAC) protocol, backwards compatible to the legacy IEEE 802.11 [11], that coordinates the transmissions among a set of relay nodes which act as helpers in a bidirectional communication. Our main contribution lies on the following:

1. Network coding techniques are used along with cooperative ARQ to enhance the system performance.

2. Less control packets - and consequently less overhead - are added in the network.

3. The contention phases are decreased, hence reducing the idle and the collision times.

4. An analytical model for the energy efficiency of the system is presented.

The rest of the paper is organized as follows. Section 2 provides the reader with the basic background on cooperative networking and outlines the related work on MAC layer protocols for both simple and network coding-based cooperative schemes in the literature. In Section 3, we introduce our proposed NCCARQ-MAC protocol along with a detailed mathematical analysis. The validation of the analytical model and the numerical results are provided in 
Section 4. Finally, Section 5 concludes the paper.

\section{Background and Related Work}

\subsection{Cooperative Communication}

In the context of cooperative communications, several schemes focused on MAC layer have already been proposed in the literature [12]-[18]. These works can be classified in two main categories: i) the cooperative ARQbased protocols and ii) the protocols that transform one-hop transmissions to multi-hop transmissions by exploiting the multi-rate capabilities of wireless systems.

\subsubsection{Cooperative ARQ-based protocols}

Forward Error Correction (FEC) and Automatic Repeat reQuest (ARQ) algorithms are two basic error control methods for data communications [19]. ARQ schemes have received considerable attention for data transmissions due to their simplicity and higher reliability, compared to FEC schemes.

Regarding the protocols falling in this category [12]-[14], the retransmissions are initiated by the destination after an erroneous packet reception. The helpers in a network are enabled to relay the original packets, as ARQ defines, using higher data rates or better channel conditions in terms of Signal-to-Noise-Ratio (SNR) values.

\subsubsection{From One-Hop to Multi-Hop Transmission via Adaptive Modulation}

By using the concept of adaptive modulation [20], mobile stations in multi-rate wireless networks assign the modulation scheme and transmission rate according to the detected Signal-to-Noise-Ratio (SNR) and the required 
transmission quality. Each modulation scheme could be further mapped to a range of SNR in a given transmission power. To achieve high transmission efficiency in wireless systems, stations select the highest available rate modulation scheme according to the detected SNR.

The protocols of this class [15]-[18] transform single one-hop transmissions to multi-hop transmissions according to the channel conditions. Specifically, the routing of the packets takes place by taking into account the channel state between the source, the relay and the destination, and therefore a multi-hop transmission may be preferred instead of the direct one.

\subsection{Cooperation and Network Coding}

Lately, there is a trend towards incorporating network coding in cooperative communications. The initial attempts for developing network codingbased cooperative communications focused on physical layer schemes [21][23]. These approaches refer to the coding gain and optimal power allocation in simple cooperative topologies, usually considering one relay or cooperation among the users.

However, the innovation of applying network coding in cooperative communications is not confined only in the physical layer. Umehara et al. [24] developed analytical models for the throughput and the delay in slotted ALOHA with network coding (S-ALOHA/NC) for single-relay multi-user wireless networks with bidirectional data flows. Recently, Munari et al. [25] introduced PHOENIX, which exploits the advanced Multiple-Input-MultipleOutput (MIMO) network coding principle to enable the relay nodes to code their own data packets along with noisy versions of packets received from other nodes. Another work that deals with the MAC layer of network coding- 
based cooperative communication was presented by Tan et al. [26]. Their proposed protocol, called CODE, exploits the benefits of both network coding and multi-rate capabilities of IEEE 802.11 Standard. Specifically, the coding of the packets takes place at the relay nodes, under two basic conditions: i) the direct link between the sender and the receiver is poor and exists one or more relay candidates that experience better link conditions and ii) the traffic is bidirectional.

\subsection{Network Coding and Energy Efficiency}

The impact of network coding in "green" communications has already started to be studied, especially in broadcast and multicast scenarios [27][30]. The recent research work that investigates the energy aspect of network coding applications, deals mostly with the network layer. Cui et al. [31] introduced CORP by using a suboptimal scheduling algorithm that exploits network coding opportunities, thus achieving a significant power saving over pure routing. More recently, Miao et al. [32] proposed an energy efficient broadcast algorithm using network coding for gradient-based routing (GBR) in wireless networks. Their proposed algorithm aims to reduce the network traffic and, consequently, the energy consumption, thus prolonging the network lifetime.

Considering the research carried out in the related fields, the main goal of our work is threefold: i) to exploit the benefits of network coding in cooperative ARQ scenarios, ii) to introduce a MAC protocol for multiple relay nodes, and iii) to study the energy efficiency of the proposed protocol from the MAC layer point of view. 


\section{Proposed Network Coding-based Cooperative ARQ (NCCARQ) MAC Protocol}

\subsection{Protocol Description}

NCCARQ-MAC has been designed to coordinate the retransmissions among a set of relays that support a bidirectional communication between pairs of nodes. The first goal of NCCARQ-MAC is to enable the IEEE 802.11 stations to request cooperation by the neighboring nodes upon an erroneous reception of a data packet. The second design goal of our proposed protocol is to allow the helper nodes to perform network coding techniques to the packets to be transmitted before relaying them.

Two fundamental requirements are needed for the efficient operation of NCCARQ-MAC: i) all nodes in the network should operate in a promiscuous mode in order to be able to listen to all ongoing transmissions and cooperate if requested, and ii) the relays should store a copy of any captured data packet (regardless of its destination address) until it is acknowledged by the intended destination.

In NCCARQ-MAC, a cooperation phase is initiated once a packet is not received correctly by the destination ${ }^{3}$. Several error detection mechanisms such as Cyclic Redundancy Code (CRC) can be applied to perform error control to the received messages. Therefore, the destination station initiates the cooperation phase by broadcasting a Request for Cooperation (RFC) message after sensing the channel idle for SIFS (Short Inter Frame Space) period

\footnotetext{
${ }^{3}$ The terms "source" and "destination" are used with regard to the initial transmission. Throughout the communication, both nodes can be source or destination of data flows.
} 
of time. This message has the form of a control packet and higher priority over regular data traffic, since data transmissions in 802.11 take place after a longer period of silence (DCF Inter Frame Space - DIFS). Furthermore, in the special but not rare case of bidirectional traffic, i.e. when the destination has a data packet for the source, the packet is broadcasted piggy-backed on the RFC message.

The stations that receive the RFC packet are potential candidates to become active relays for the communication process. Therefore, the relay set is formed upon the reception of the RFC and the participants (helpers) get ready to forward their information. Since the partners have already stored the packets destined both to the destination (so called cooperative packet) and to the source (so called piggy-backed packet), they create a new coded packet by combining the two existing data packets, using the XOR method. Accordingly, the active relays will try to get access to the channel in order to persistently transmit the network coded (NC) packet. Once the source and the destination receive the $\mathrm{NC}$ packet from the relay, they are able to decode it and extract the original data packets. Subsequently, they acknowledge the received data packet by transmitting the respective ACK, thus terminating the cooperation phase. Receiving the acknowledgment packets, the relays are informed that the particular communication has been completed, hence becoming able to erase the packets of their buffers. In case that either one or both of the received coded packets can not be decoded after a certain maximum cooperation timeout due to transmission errors, the relays are obliged to forward again the NC packet. A simple scenario that is subject to the principles of NCCARQ-MAC is depicted in Figure 1. 


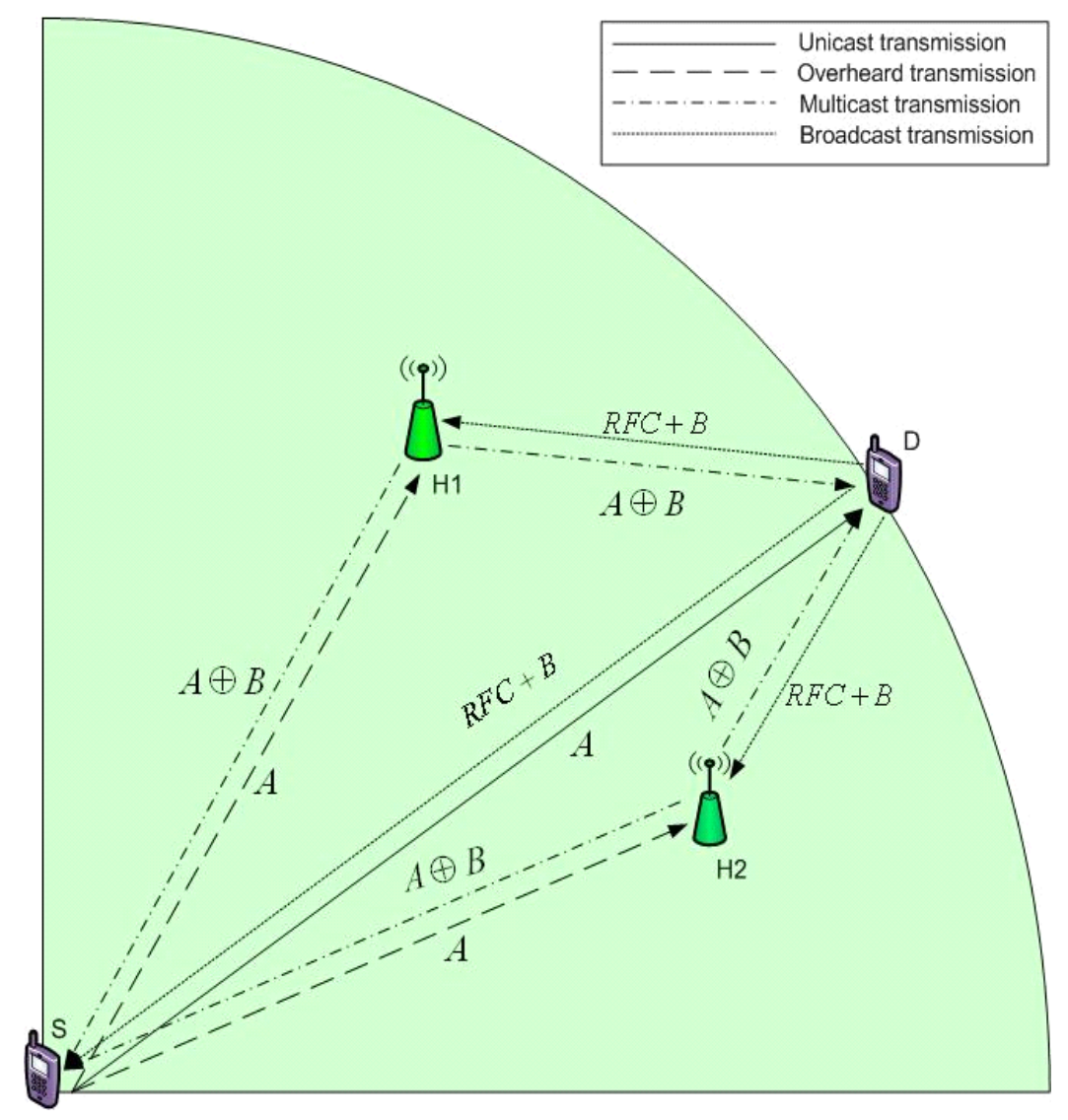

Figure 1: General idea of NCCARQ-MAC operation

As it has been already mentioned, NCCARQ-MAC is backward compatible with IEEE 802.11 Standard, as it uses the same frame structure and follows the same principles with the Standard. However, some modifications have been made in order for the protocol to efficiently exploit the advantages of both cooperative and network coding techniques:

1. There exist ACK packets for the transmission of the $\mathrm{NC}$ packet to guarantee a reliable multicast communication scheme.

2. For bidirectional traffic, the data packets are transmitted along with 
the RFC packets, without taking part in the contention phase.

3. Since the subnetwork formed by the relay set operates in saturated conditions, it is necessary to execute a backoff mechanism at the beginning of the cooperation phase to minimize the probability of a certain initial collision.

\subsection{Operational Example}

Let us provide a more accurate step-by-step description of the working operation of NCCARQ-MAC for the network scenario in Figure 1. A simple network topology with four stations is considered, all of them in the transmission range of each other. A source station $(S)$ transmits a data packet $(A)$ to a destination station $(D)$ that does also have a packet $(B)$ destined to the source station. Furthermore, there are two helper nodes $\left(H_{1}\right.$ and $\left.H_{2}\right)$ that support this particular bidirectional communication. Our protocol has been designed to handle an arbitrary number of relays and, hence, the two-relay network topology in Figure 1 is given only for illustrative purposes.

In detail, the protocol works as follows (see Figure 2):

1. At instant $t_{1}$, station $S$ sends the data packet $A$ to station $D$.

2. Upon reception, at instant $t_{2}$, station $D$ fails to demodulate the data packet, thus broadcasting an $R F C$ packet asking for cooperation by the neighboring stations ( $H_{1}$ and $H_{2}$ in this example) along with the data packet $B$, destined to the station $S$.

3. The reception of the $R F C\left(t_{3}\right)$ triggers the stations $H_{1}$ and $H_{2}$ to become active relays and set up their backoff counters $\left(C W_{1}\right.$ and $C W_{2}$, respectively) in order to participate in the contention phase. 
4. At instant $t_{4}$, the backoff counter of $H_{1}$ expires and $H_{1}$ transmits the coded packet $A \oplus B$ to the nodes $S$ and $D$ simultaneously.

5. At instant $t_{5}$, the station $D$ retrieves the original packet $A$ and sends back an $A C K$ packet since it is able to decode properly the XOR-ed packet.

6. At instant $t_{6}$, the node $S$ acknowledges the packet $B$ since it is able to decode properly the coded packet $A \oplus B$.

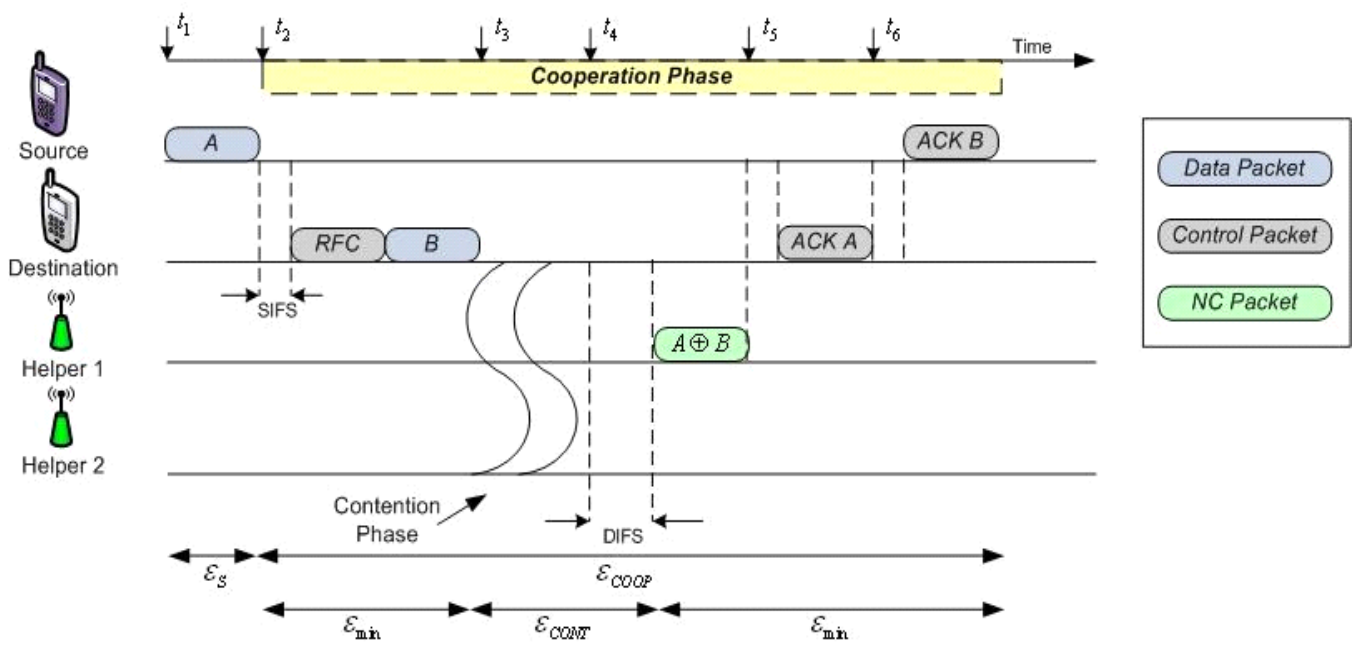

Figure 2: NCCARQ-MAC example of frame sequence

\subsection{Protocol Analysis}

\subsubsection{Delay Analysis}

The network coding techniques in our proposed scheme imply the simultaneous transmission of more than one packet in the network. Therefore, we analytically estimate the expected time needed for two packets to be exchanged under the NCCARQ-MAC protocol. 
The total time elapsed from the initial transmission until the correct reception at the destinations can be defined as:

$$
\mathbf{E}\left[T_{\text {total }}\right]=\mathbf{E}\left[T_{S}\right]+\mathbf{E}\left[T_{C O O P}\right]
$$

where $\mathbf{E}\left[T_{S}\right]$ represents the average time for the direct transmission of a single data packet from the source to destination and $\mathbf{E}\left[T_{C O O P}\right]$ corresponds to the average time required for a cooperative transmission via relays to be completed.

Since $\mathbf{E}\left[T_{S}\right]$ has a value that is easy to be estimated depending on the network's configuration, we focus our analysis on the term $\mathbf{E}\left[T_{C O O P}\right]$ in order to derive a closed-form expression for the system delay. The average time spent during the cooperation phase can be defined as:

$$
\mathbf{E}\left[T_{C O O P}\right]=\mathbf{E}\left[T_{\min }\right]+\mathbf{E}\left[T_{C O N T}\right]
$$

where $\mathbf{E}\left[T_{\min }\right]$ is the minimum average delay in case of perfect scheduling among the relays, i.e. contention-free scheme. On the other hand, the term $\mathbf{E}\left[T_{C O N T}\right]$ is used to denote the additional delay that is caused due to the contention phase which has been adopted in our protocol to minimize the probability of collisions.

In general, the expected number of retransmissions $(\mathbf{E}[r])$ that are required in order to properly demodulate the coded packet at the destination nodes is directly connected with the packet error rate between the relays and the destination $\left(P E R_{R \rightarrow D}\right)$. However, in our scheme, two packets are sent at the same time via different channels and, as a result, the number of retransmissions can be expressed as [33]:

$$
\mathbf{E}[r]=\frac{1+\frac{\left(1-P E R_{1}\right) \cdot P E R_{2}}{1-P E R_{2}}+\frac{\left(1-P E R_{2}\right) \cdot P E R_{1}}{1-P E R_{1}}}{1-P E R_{1} \cdot P E R_{2}}
$$


where $P E R_{1}$ and $P E R_{2}$ represent the $P E R_{R \rightarrow S}$ and the $P E R_{R \rightarrow D}$, respectively.

Therefore, the term $\mathbf{E}\left[T_{\min }\right]$ can be calculated as:

$$
\begin{aligned}
& \mathbf{E}\left[T_{\text {min }}\right]=T_{S I F S}+T_{R F C}+T_{B}+T_{O N C}+\mathbf{E}[r] \cdot\left(T_{D I F S}+T_{A \oplus B}+T_{S I F S}\right)+ \\
& +T_{A C K}+T_{S I F S}+T_{A C K}
\end{aligned}
$$

where $T_{R F C}$ and $T_{A C K}$ are the transmission times for the $R F C$ and the $A C K$ packet, respectively. Furthermore, $T_{A \oplus B}$ is the time required to retransmit a coded packet, while $T_{O N C}$ is the time that a relay needs for applying network coding techniques. Finally, $T_{S I F S}$ and $T_{D I F S}$ is the duration of a SIFS and a DIFS silence period, respectively.

Moreover, the term $\mathbf{E}\left[T_{C O N T}\right]$ can be defined as:

$$
\mathbf{E}\left[T_{C O N T}\right]=\mathbf{E}[r] \cdot \mathbf{E}\left[T_{c}\right]
$$

where $\mathbf{E}\left[T_{c}\right]$ represents the average time required to transmit a $\mathrm{NC}$ packet during the contention phase among all the relays. The theoretical estimation of $\mathbf{E}\left[T_{c}\right]$ is associated to Appendix A. Applying this analysis, the formula (5) can be rewritten as:

$$
\mathbf{E}\left[T_{C O N T}\right]=\mathbf{E}[r] \cdot\left(\frac{1}{p_{s}}-1\right)\left[\left(\frac{p_{i}}{1-p_{s}}\right) \sigma+\left(\frac{p_{c}}{1-p_{s}}\right) T_{c o l}\right] .
$$

Hence, we are able to derive a closed-form formula and compute the total delay for two packets to be exchanged in the system by exploiting the equations (1), (2), (4) and (6).

\subsubsection{Throughput Analysis}

The total throughput of the network can be defined as the sum of the throughput produced by the successful direct transmissions from the source 
plus the throughput derived by the cooperation phase after erroneous packet receptions. This can be mathematically expressed as:

$$
\mathbf{E}\left[S_{\text {total }}\right]=\mathbf{E}\left[S_{S}\right]+\mathbf{E}\left[S_{C O O P}\right]
$$

where

$$
\mathbf{E}\left[S_{S}\right]=\left(1-P E R_{S \rightarrow D}\right) \cdot \frac{\mathbf{E}[P]}{\mathbf{E}\left[T_{S}\right]}
$$

and

$$
\mathbf{E}\left[S_{C O O P}\right]=2 \cdot P E R_{S \rightarrow D} \cdot \frac{\mathbf{E}[P]}{\mathbf{E}\left[T_{\text {total }}\right]}
$$

In the above expressions, the parameters $\mathbf{E}\left[T_{S}\right]$ and $\mathbf{E}\left[T_{\text {total }}\right]$ have already been defined in Section 3.3.1. Furthermore, the packet error rate between the source and the destination is given by $P E R_{S \rightarrow D}$, while $\mathbf{E}[P]$ denotes the average packet payload. In this point, it must be clarified that the coefficient 2 in formula (9) is mandatory, since two packets are delivered in each particular successful retransmission.

Thus, having obtained a closed-form expression for $\mathbf{E}\left[T_{\text {total }}\right]$ and since $\mathbf{E}[P], \mathbf{E}\left[T_{S}\right]$ and $P E R_{S \rightarrow D}$ are known parameters, we are able to compute the theoretical system throughput.

\subsubsection{Energy Performance Analysis}

Having analyzed the operation of the proposed NCCARQ-MAC protocol, we derive a closed-form expression that describes the average energy consumption in the network:

$$
\mathbf{E}\left[\mathcal{E}_{\text {total }}\right]=\mathbf{E}\left[\mathcal{E}_{S}\right]+\mathbf{E}\left[\mathcal{E}_{C O O P}\right]
$$


where $\mathbf{E}\left[\mathcal{E}_{C O O P}\right]$ and $\mathbf{E}\left[\mathcal{E}_{S}\right]$ represent the average energy consumption during the cooperative phase and the initial transmission from the source, respectively. The term $\mathbf{E}\left[\mathcal{E}_{C O O P}\right]$ could be further expressed as:

$$
\mathbf{E}\left[\mathcal{E}_{C O O P}\right]=\mathbf{E}\left[\mathcal{E}_{m i n}\right]+\mathbf{E}\left[\mathcal{E}_{C O N T}\right]
$$

where $\mathbf{E}\left[\mathcal{E}_{\text {min }}\right]$ denotes the energy waste in a perfect scheduled cooperative phase and $\mathbf{E}\left[\mathcal{E}_{C O N T}\right]$ is the energy consumed during the contention phase (i.e. idle and collided slots). The particular energy consumptions are depicted in Figure 2 for illustrative purposes.

In order to clarify the above equations, we try to compute each term analytically. We consider three different modes:

1. Transmission mode, when the node is transmitting data/control packets.

2. Reception mode, when the node is receiving data/control packets.

3. Idle mode, when the node is sensing the medium without performing any action.

The power levels associated to each mode are $P_{T}, P_{R}$ and $P_{I}$, respectively. Furthermore, the relationship between energy and power is given by $\mathcal{E}=P \cdot t$, where the terms $\mathcal{E}, P$ and $t$ represent the energy, the power and the time, respectively.

We recall that the network consists of a source, a destination and a set of $n$ relays. Therefore, considering the network topology, we have:

$$
\mathbf{E}\left[\mathcal{E}_{S}\right]=P_{T} \cdot T_{A}+(n+1) \cdot P_{R} \cdot T_{A}
$$




$$
\begin{aligned}
& \mathbf{E}\left[\mathcal{E}_{m i n}\right]=(n+2) \cdot P_{I} \cdot T_{S I F S}+P_{T} \cdot\left(T_{R F C}+T_{B}\right)+(n+1) \cdot P_{R} \cdot\left(T_{R F C}+T_{B}\right)+(n+2) \cdot P_{I} \cdot T_{O N C}+ \\
& +\mathbf{E}[r] \cdot\left((n+2) \cdot P_{I} \cdot T_{D I F S}+P_{T} \cdot T_{A \oplus B}+2 \cdot P_{R} \cdot T_{A \oplus B}+(n-1) \cdot P_{I} \cdot T_{A \oplus B}+(n+2) \cdot P_{I} \cdot T_{S I F S}\right)+ \\
& +2 \cdot P_{T} \cdot T_{A C K}+2 \cdot(n+1) \cdot P_{R} \cdot T_{A C K}+(n+2) \cdot P_{I} \cdot T_{S I F S}
\end{aligned}
$$

where the definitions of the parameters can be found in Section 3.3.1.

The above equations (12)-(13) are based on the following principles:

- All stations remain idle during the SIFS, DIFS and $T_{O N C}$ times.

- The relays that lose in the contention phase turn in idle mode.

- When a station transmits a packet (control or data), the rest of the stations are in promiscuous mode, thus capturing the packets.

Computing the energy consumed during the contention phase constitutes one of the most challenging parts in this analytical model. The total energy waste during this phase derives from the energy spent during both the idle slots and the collisions. Let us start by defining that:

$$
\mathbf{E}\left[\mathcal{E}_{C O N T}\right]=\mathbf{E}[r] \cdot \mathbf{E}\left[\mathcal{E}_{C}\right]
$$

where $\mathbf{E}\left[\mathcal{E}_{C}\right]$ represents the average energy required to transmit a NC packet during the contention phase among all the relays. In order to calculate the energy consumed during the collisions, we have to estimate the average number of stations that transmit a packet simultaneously. The probability $p_{k}$ that exactly $k$ stations are involved in a collision is:

$$
p_{k}=\frac{\left(\begin{array}{l}
n \\
k
\end{array}\right) \tau^{k}(1-\tau)^{n-k}}{p_{c}}
$$


where $\tau$ and $p_{c}$ are defined in Appendix A.

Therefore, the expected number $\mathbf{E}[K]$ of stations that are involved in a collision is:

$$
\mathbf{E}[K]=\sum_{k=2}^{n} k \cdot p^{k}=\sum_{k=2}^{n} k \cdot \frac{\left(\begin{array}{l}
n \\
k
\end{array}\right) \tau^{k}(1-\tau)^{n-k}}{p_{c}}
$$

During the idle slots, all the stations in the network remain idle. On the other hand, during the collisions, more than one relay is in transmission mode, two stations (the source and the destination) are in reception mode, while the rest of the relays are in idle mode. Considering the probabilities that we have derived regarding the contention phase $\left(p_{c}, p_{i}\right)$, the above assumptions can mathematically be expressed as:

$\mathbf{E}\left[\mathcal{E}_{C}\right]=p_{i} \cdot\left((n+2) \cdot P_{I} \cdot \sigma\right)+p_{c} \cdot\left(\mathbf{E}[K] \cdot P_{T} \cdot T_{c o l}+2 \cdot P_{R} \cdot T_{c o l}+(n-\mathbf{E}[K]) \cdot P_{I} \cdot T_{c o l}\right)$

where all the parameters have already been defined. Thus, combining the equations (10)-(14) and (17), we are able to estimate the total amount of the energy that is consumed in our protocol.

\section{Performance Evaluation}

In order to validate our analysis and further evaluate the performance of NCCARQ-MAC we have developed a time-driven $\mathrm{C}++$ simulator that executes the rules of the protocol. In the following subsections we present the simulation set up along with the results of our experiments. 


\subsection{Simulation Scenario}

The network under simulation consists of a pair of transmitter-receiver (both nodes transmit and receive data) and a set of relay nodes that facilitate the communication, all of them in the transmission range of each other. The relay set includes five (5) nodes, each of them implementing a backoff counter starting with a congestion window $\left(C W_{\min }\right)$ equal to 32 . Additionally, the relay nodes are capable of performing network coding techniques to their buffered packets before relaying them. In order to focus on the impact of both network coding and cooperative communication, we have made the following assumptions:

1. The traffic is bidirectional, i.e. the destination node has always a packet destined back to the source node.

2. Original transmissions from source to destination are always received with errors $\left(P E R_{S \rightarrow D}=1\right)$, thus initiating a cooperative phase.

3. The channel between the source and the destination is error symmetric, i.e. $P E R_{S \rightarrow D}=P E R_{D \rightarrow S}$.

4. The channel between the source and the relays is error free, i.e. $P E R_{S \rightarrow R}=0$.

5. The relay nodes transmit in a common transmission rate.

The configuration parameters of the network have been selected according to the IEEE 802.11g [11] physical layer. The time for applying network coding $\left(T_{O N C}\right)$ to the data packets is considered to be negligible, since the coding takes place between only two packets. Ebert et al. [34] have measured the power consumption of a wireless interface during the transmission and reception phase. Based on their work, we have chosen the following power levels for our scenarios: $P_{T}=1900 \mathrm{~mW}, P_{R}=P_{I}=1340 \mathrm{~mW}$. The value of 
$P_{T}$ has been selected as an average value of transmission consumed power, since it varies according to the Radio Frequency (RF) power level. The simulation parameters are summarized in Table 1.

Table 1: System Parameters

\begin{tabular}{|c|c|c|c|c|c|}
\hline Parameter & Value & Parameter & Value & Parameter & Value \\
\hline PacketLength & 1500 bytes & $R F C$ & 14 bytes & $P E R_{S \rightarrow R}$ & 0 \\
\hline MAC Header & 34 bytes & $A C K$ & 14 bytes & $P_{T}$ & $1900 \mathrm{~mW}$ \\
\hline PHY Header & $96 \mu \mathrm{sec}$ & $S I F S$ & $10 \mu \mathrm{sec}$ & $P_{R}$ & $1340 \mathrm{~mW}$ \\
\hline$C W_{\text {min }}$ & 32 & $D I F S$ & $50 \mu \mathrm{sec}$ & $P_{I}$ & $1340 \mathrm{~mW}$ \\
\hline
\end{tabular}

Furthermore, we consider three different scenarios (Table 2) with regard to the SNR between the original source and the destination. The control packets are transmitted always at the rate of $6 \mathrm{Mb} / \mathrm{s}$, while the transmission rate for the data packets is 6,24 and $54 \mathrm{Mb} / \mathrm{s}$ for low, medium and high SNR values, respectively. On the other hand, assuming that the relays are placed between the source and the destination, the transmission rates in all scenarios have been selected equal to 6 and $54 \mathrm{Mb} / \mathrm{s}$ for control and data packets, respectively. Furthermore, the network operates under saturated conditions, i.e. the nodes have always packets to send in their buffers.

In order to evaluate our approach, we compare our scheme with a simple cooperative ARQ scheme (so called CARQ-MAC), where the bidirectional communication takes place in two steps. In the first step, the source sends a packet to the destination and, upon the erroneous reception the destina- 
Table 2: Simulation Scenarios

\begin{tabular}{|l|l|l|l|l|}
\hline $\begin{array}{l}\text { SNR } \\
\text { (S-D) }\end{array}$ & $\begin{array}{l}\text { Source } \\
\text { Control } \\
\text { Rate }\end{array}$ & $\begin{array}{l}\text { Source } \\
\text { Data Rate }\end{array}$ & $\begin{array}{l}\text { Relay } \\
\text { Control } \\
\text { Rate }\end{array}$ & $\begin{array}{l}\text { Relay } \\
\text { Data Rate }\end{array}$ \\
\hline Low & $6 \mathrm{Mb} / \mathrm{s}$ & $6 \mathrm{Mb} / \mathrm{s}$ & $6 \mathrm{Mb} / \mathrm{s}$ & $54 \mathrm{Mb} / \mathrm{s}$ \\
\hline Medium & $6 \mathrm{Mb} / \mathrm{s}$ & $24 \mathrm{Mb} / \mathrm{s}$ & $6 \mathrm{Mb} / \mathrm{s}$ & $54 \mathrm{Mb} / \mathrm{s}$ \\
\hline High & $6 \mathrm{Mb} / \mathrm{s}$ & $54 \mathrm{Mb} / \mathrm{s}$ & $6 \mathrm{Mb} / \mathrm{s}$ & $54 \mathrm{Mb} / \mathrm{s}$ \\
\hline
\end{tabular}

tion broadcasts the RFC packet, thus triggering the relays to retransmit the packet. In the second step, the destination transmits its own packet to the source and the same procedure as in the first step is repeated, thus consuming valuable network resources. In both steps, the relays take part in the contention phase in order to access the medium and transmit their packets.

The metrics we use to evaluate the QoS performance of our protocol are the delay and the throughput, as they have been defined in the Sections 3.3.1 and 3.3.2, respectively. Moreover, in order to evaluate the energy performance of our proposed protocol we use the energy efficiency metric [35]. It is denoted by $\eta$, defined as:

$$
\eta=\frac{\text { total amount of useful data delivered (bits) }}{\text { total energy consumed (Joule) }}
$$

Before proceeding to the simulation results, it is worth mentioning that the definition of equation (18) inherently implies that network coding benefits the energy efficiency of a protocol, as the number of the delivered bits increases by combining multiple data packets. 


\subsection{Performance Results}

Figure 3 presents the throughput performance in the three scenarios described above for various values of $P E R_{R \rightarrow D}$. First, we can see that the theoretical and the simulation results are almost perfectly matched, thus verifying our analysis. Comparing with simple cooperative schemes which have only the advantage of spatial diversity without any network coding capabilities, we can achieve a throughput enhancement up to $80 \%$. It is also worth noticing that NCCARQ-MAC outperforms CARQ-MAC even for worse SNR scenarios. To clarify, observing the achievable throughput in NCCARQ-MAC for the medium SNR scenario we can see that it clearly outperforms CARQ-MAC under the high SNR scenario.

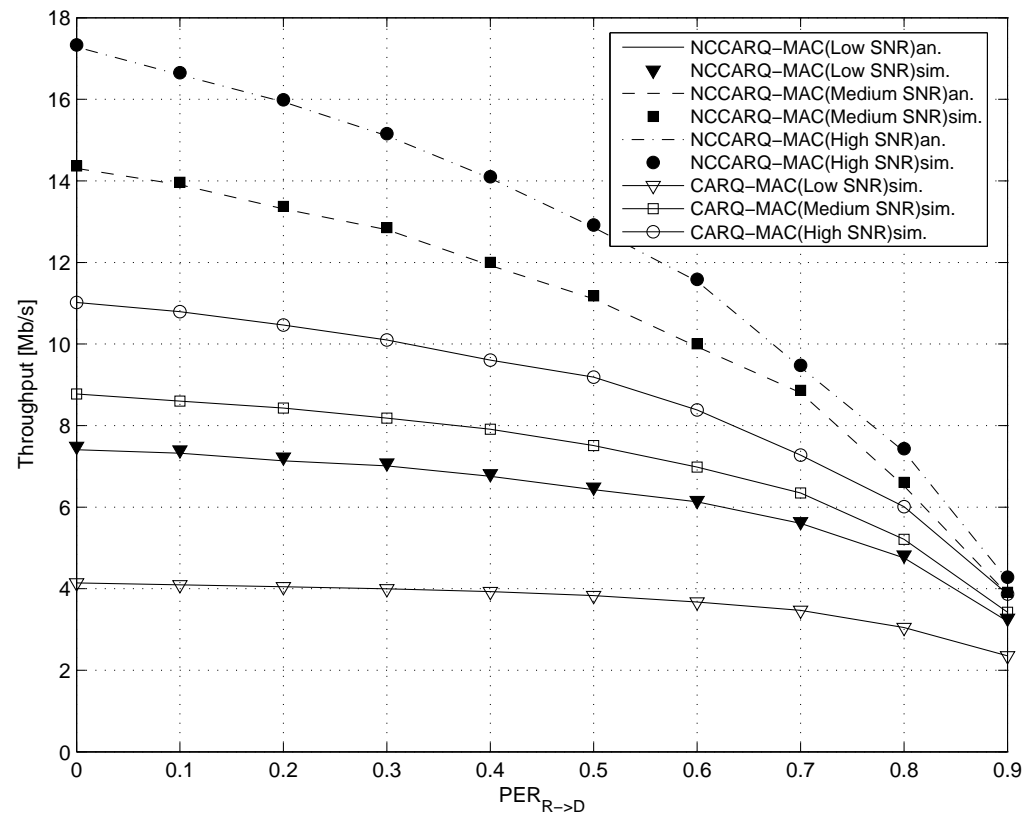

Figure 3: System Throughput (NCCARQ-MAC vs. CARQ-MAC)

Figure 4 shows the throughput performance with regard to the minimum 
congestion window $\left(C W_{\text {min }}\right)$ of the wireless nodes. In this specific case (low SNR scenario), there are ten relays in the network (in total twelve nodes including the source and the destination) and we assume that $P E R_{R \rightarrow D}=0.5$, i.e. two retransmissions on average are needed for each NC packet to be delivered properly $(\mathbf{E}[r]=2)$. As it was expected, the two curves exhibit similar behavior, achieving maximum throughput for $C W \min =64$. However, a useful conclusion that can be derived from the figure is that NCCARQ-MAC outperforms CARQ-MAC independently of the initial $C W$.

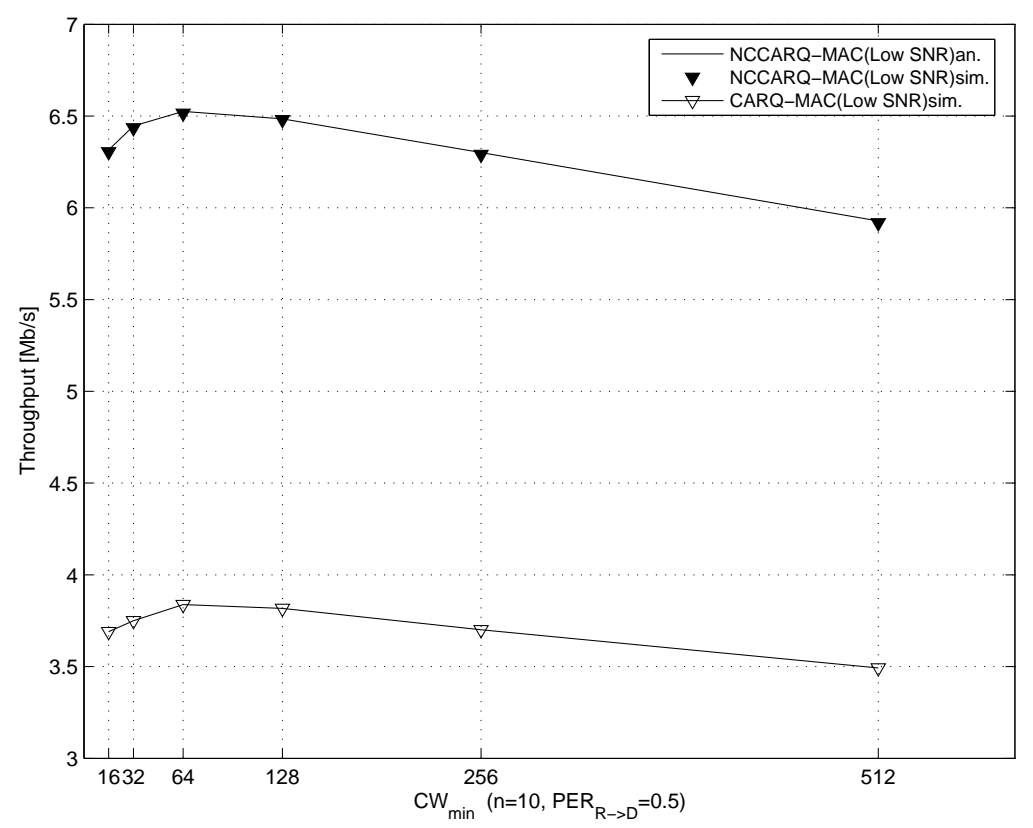

Figure 4: System Throughput (NCCARQ-MAC vs. CARQ-MAC) $(\mathrm{n}=10, \mathbf{E}[r]=2)$

Figure 5 presents the packet delay in both Network Coding-based and simple Cooperative ARQ MAC protocols. In this point, we must recall that two packets are delivered to their respective destinations in each transmission cycle of NCCARQ-MAC. Hence, in order to be accurate, we compare 
the delay in NCCARQ-MAC with the time required for two packets to be exchanged in CARQ-MAC.

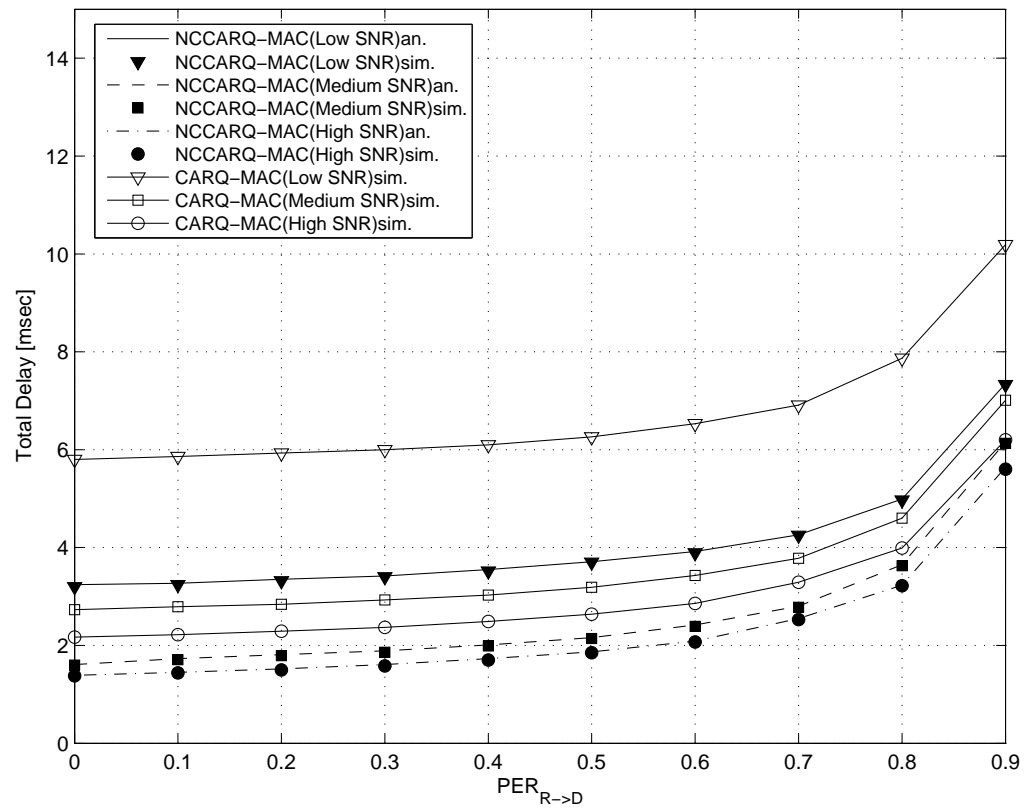

Figure 5: Two-Packet Delay (NCCARQ-MAC vs. CARQ-MAC)

As it can be observed, we can achieve significantly lower packet delay by applying network coding techniques. For example, considering the low SNR scenario, the average time that is required for two packets to be transmitted using CARQ-MAC is 5.9 msec in error-free channels where one retransmission is necessary, reaching up to $7.9 \mathrm{msec}$ when $P E R_{R \rightarrow D}=0.8$, i.e. five retransmissions are required on average. On the other hand, the delay values in NCCARQ-MAC are 3.3 and $5 \mathrm{msec}$, for one and five retransmissions, respectively. This difference can be rationally explained considering the operation of NCCARQ-MAC, where some data packets are sent to the relay attached to the RFC message, thus avoiding the erroneous channel. Furthermore, in 
our proposed scheme we manage to reduce the backoff phases by sending two packets simultaneously, while in simple cooperative protocols the relays have to participate in the contention phase for each packet that has to be retransmitted. Therefore, we are able to enhance the packet delay, since the time that is spent in idle slots and collisions is significantly reduced, especially as the PER between the relays and the destination grows.

Figure 6 shows that the simulation results verify our analysis regarding the energy performance. Comparing our proposed network coding-based scheme with simple cooperative protocols for different PER (and consequently different number of retransmissions) between the relays and the destinations, we observe that our scheme is more energy efficient than CARQMAC, since more bits are delivered over the same amount of energy consumed. Keeping constant the data packet payload (1500 bytes) the energy efficiency of NCCARQ-MAC decreases as the packet error rate between the relays and the destination grows. However, the gain compared to simple cooperative schemes remains steadily over $60 \%$ for reasonable values of $P E R$ (i.e. $P E R<0.7)$.

In particular, comparing the two protocols under the same conditions (i.e. when both schemes operate under similar SNR values), we observe that NCCARQ-MAC outperforms CARQ-MAC in all cases. Furthermore, it is worth mentioning that network coding is proved to be beneficial for the system when the communication takes place under bad channel conditions. To make it clear, observing the energy performance of the NCCARQ-MAC for the medium SNR scenario we can see that it clearly outperforms the CARQ-MAC under the high SNR scenario. However, in all cases, the gain 


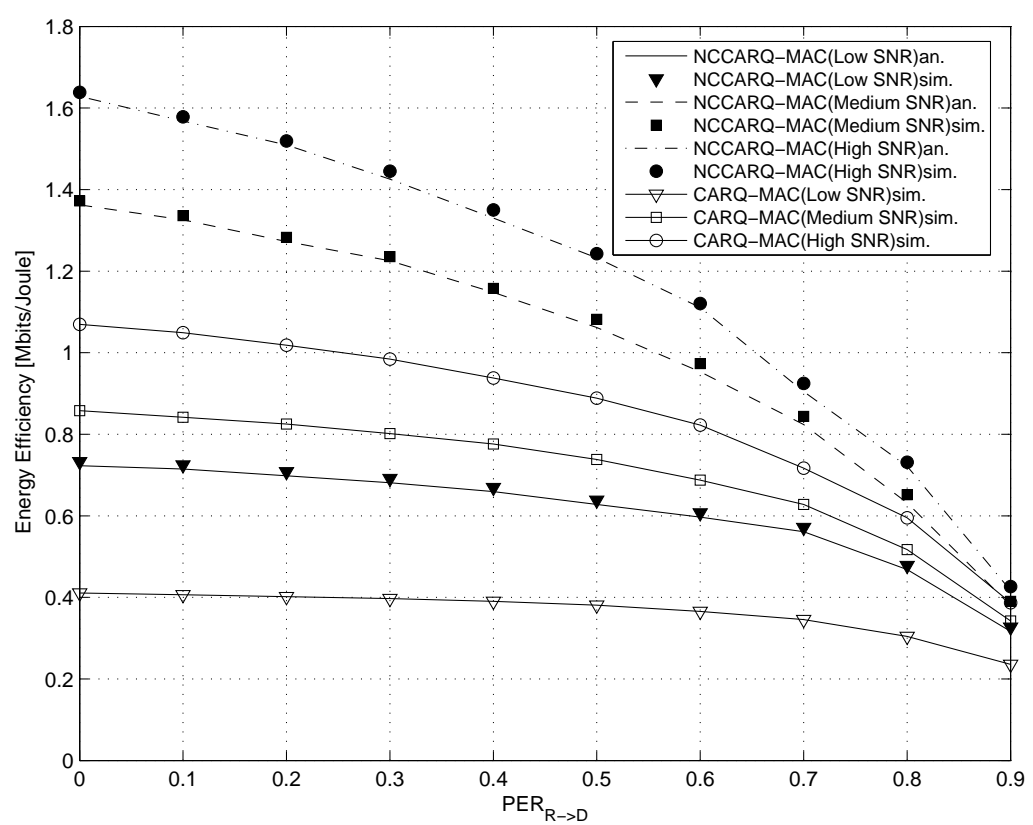

Figure 6: Energy Efficiency (NCCARQ-MAC vs. CARQ-MAC)

of network coding becomes asymptotically negligible as $P E R_{R \rightarrow D} \rightarrow 1$.

\section{Conclusion}

In this paper, a novel network coding-based cooperative ARQ (NCCARQMAC) scheme for wireless networks was presented. Compared to simple cooperative ARQ protocols, the proposed solution improves up to $80 \%$ the energy efficiency of the system without compromising the offered QoS in terms of throughput and delay. Our future work will be focused on the impact of realistic physical layer on our protocol. 


\section{Appendix A. $\mathrm{E}\left[\boldsymbol{T}_{c}\right]$ Analysis}

In order to analytically compute the $\mathbf{E}\left[T_{c}\right]$ value we need to model the backoff counter of each of the relays with the Markov Chain introduced by Bianchi in [36] (Figure A.7), since the formed subnetwork acts as a saturated IEEE 802.11 ad hoc network despite the modifications in the access rules. According to this model, the probability $\tau$ that a station transmits in a randomly chosen slot, is given by:

$$
\tau=\sum_{i=1}^{m} b_{i, 0}=\frac{b_{0,0}}{1-p}=\frac{2(1-2 p)}{(1-2 p)(W+1)+p W\left(1-(2 p)^{m}\right)}
$$

where

$$
b_{0,0}=\frac{2(1-2 p)(1-p)}{(1-2 p)(W+1)+p W\left(1-(2 p)^{m}\right)}
$$

and the probability of a collision $p$ as a function of $\tau$ is given by:

$$
p=1-(1-\tau)^{n-1}
$$

In the formulas (A.1)-(A.2), $b_{i, k}$ represents the steady state probability of the state $\{i, k\}, W$ is the size of the congestion window, $m$ denotes the number of the backoff stages and $n$ corresponds to the number of the relays in the network.

Furthermore, the probability that at least one relay attempts to transmit can be expressed as:

$$
p_{t r}=1-(1-\tau)^{n}
$$

and the probability of a successful transmission, i.e. one station transmits conditioned on the fact that at least one station transmits is given by:

$$
p_{s \mid t r}=\frac{n \tau(1-\tau)^{n-1}}{1-(1-\tau)^{n}}
$$




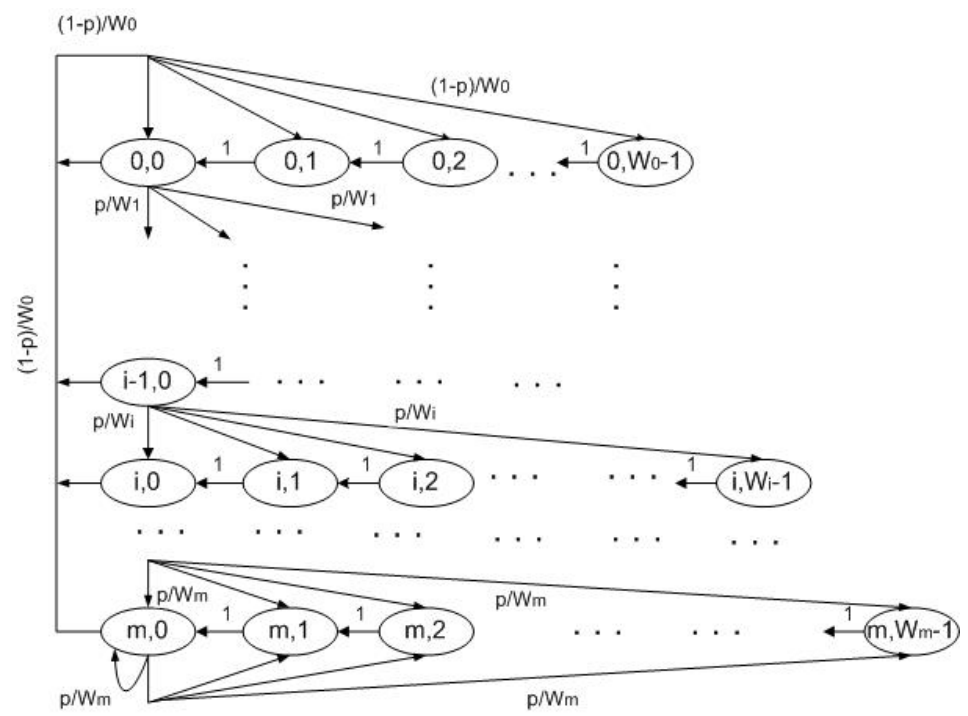

Figure A.7: The 2-dimensional Markov model state transition diagram

In addition, the probabilities of having an idle $\left(p_{i}\right)$, successful $\left(p_{s}\right)$ or collided $\left(p_{c}\right)$ slot can be written as:

$$
\begin{gathered}
p_{i}=1-p_{t r} \\
p_{s}=p_{t r} \cdot p_{s \mid t r} \\
p_{c}=p_{t r} \cdot\left(1-p_{s \mid t r}\right)
\end{gathered}
$$

Considering the above probabilities, and given that the average number of slots that we have to wait before having a successful transmission can be represented as:

$$
\mathbf{E}[N]=\sum_{k=0}^{\infty} k\left(1-p_{s}\right)^{k} p_{s}=\frac{1}{p_{s}}-1,
$$

the total contention time can be written as

$$
\mathbf{E}\left[T_{c}\right]=\mathbf{E}[N] \cdot \mathbf{E}\left[T_{\text {slot } \mid \text { non_successful_slot }}\right] .
$$


Applying Bayes' theorem we are able to estimate the average duration of a slot, given that the specific slot is either idle or collided:

$$
\mathbf{E}\left[T_{\text {slot } \mid \text { non_successful_slot }}\right]=\left(\frac{p_{i}}{1-p_{s}}\right) \sigma+\left(\frac{p_{c}}{1-p_{s}}\right) T_{c o l}
$$

with $\sigma$ representing the duration of an idle slot, while $T_{c o l}$ corresponds to the time of a collision and in our scheme is equal to:

$$
T_{c o l}=T_{D I F S}+T_{A \oplus B}+T_{S I F S} .
$$

Hence, equation (A.10) is written as:

$$
\mathbf{E}\left[T_{c}\right]=\left(\frac{1}{p_{s}}-1\right) \cdot\left[\left(\frac{p_{i}}{1-p_{s}}\right) \sigma+\left(\frac{p_{c}}{1-p_{s}}\right) T_{c o l}\right] .
$$

\section{References}

[1] T. M. Cover and A. A. E. Gamal, "Capacity Theorems for the Relay Channel", IEEE Trans. Info. Theory, vol. 25, no. 5, Sept. 1979, pp. 57284.

[2] R. Ahlswede, Ning Cai, S.-Y.R. Li, R.W. Yeung, "Network Information Flow", IEEE Transactions on Information Theory, vol.46, no.4, pp.12041216, Jul 2000

[3] S.-Y. R. Li, R. W. Yeung, Ning Cai, "Linear network coding", IEEE Transactions on Information Theory, vol.49, no.2, pp.371-381, Feb. 2003

[4] M. Ghaderi, D. Towsley, J. Kurose, "Reliability Gain of Network Coding in Lossy Wireless Networks", INFOCOM 2008. The 27th Conference on Computer Communications. pp.2171-2179, 13-18 April 2008 
[5] R. Ahlswede, H. Aydinian, "On Error Control Codes for Random Network Coding", Workshop on Network Coding, Theory, and Applications (NetCod) 2009. pp.68-73, 15-16 June 2009

[6] Ning Cai, "Valuable Messages and Random Outputs of Channels in Linear Network Coding", IEEE International Symposium on Information Theory (ISIT), pp.413-417, June 28-July 3, 2009

[7] H. Bahramgiri, F. Lahouti, "Robust Network Coding Against Path Failures", IET Communications, vol.4, no.3, pp.272-284, Feb. 122010

[8] S. Katti, H. Rahul, Wenjun Hu, D. Katabi, M. Medard, J. Crowcroft, "XORs in the Air: Practical Wireless Network Coding", IEEE/ACM Transactions on Networking, vol.16, no.3, pp.497-510, June 2008

[9] A. Argyriou, "Wireless Network Coding with Improved Opportunistic Listening", IEEE Transactions on Wireless Communications, vol.8, no.4, pp.2014-2023, April 2009

[10] J. Zhang, Y. P. Chen, I. Marsic, "MAC-layer proactive mixing for network coding in multi-hop wireless networks", Computer Networks, Volume 54, Issue 2, Wireless Multi-Hop Networking for Infrastructure Access, 15 February 2010, pp. 196-207, ISSN 1389-1286, DOI: 10.1016/j.comnet.2009.04.013.

[11] IEEE 802.11g WG, Part 11: Wireless LAN Medium Access Control (MAC) and Physical Layer (PHY) specifications - Amendment 4: Further Higher Data Rate Extension in the 2.4 GHz Band, June 2003. 
[12] Xin He, F. Y. Li, "An Automatic Cooperative Retransmission MAC Protocol in Wireless Local Area Networks", European Wireless Conference (EW) 2009. pp.228-233, 17-20 May 2009

[13] Kejie Lu, Shengli Fu, Yi Qian, "Increasing the Throughput of Wireless LANs Via Cooperative Retransmission", IEEE Global Telecommunications Conference (GLOBECOM), 2007. pp.5231-5235, 26-30 Nov. 2007

[14] J. Alonso-Zarate, E. Kartsakli, Ch. Verikoukis, and L. Alonso, "Persistent RCSMA: A MAC Protocol for a Distributed Cooperative ARQ Scheme in Wireless Networks", EURASIP Journal on Advances in Signal Processing, vol. 2008, Article ID 817401, 13 pages, 2008. doi:10.1155/2008/817401

[15] Z. Hu, C.-K. Tham, "CCMAC: Coordinated cooperative MAC for wireless LANs", Computer Networks, Volume 54, Issue 4, Advances in Wireless and Mobile Networks, 19 March 2010, pp. 618-630, ISSN 1389-1286, DOI: 10.1016/j.comnet.2010.02.001.

[16] Tao Guo, R. Carrasco, "CRBAR: Cooperative Relay-based Auto Rate MAC for Multirate Wireless Networks", IEEE Transactions on Wireless Communications, vol.8, no.12, pp.5938-5947, December 2009

[17] Xing-Jian Zhu, Geng-Sheng Kuo, "Cooperative MAC Scheme for MultiHop Multi-Channel Wireless Mesh Networks," Vehicular Technology Conference (VTC) 2008-Fall. IEEE 68th , pp.1-6, 21-24 Sept. 2008

[18] Chang-Yeong Oh, Tae-Jin Lee, "MAC Protocol using Cooperative Active Relays in Multi-Rate Wireless LANs", IFIP International Confer- 
ence on Wireless and Optical Communications Networks (WOCN) 2009. pp.1-6, 28-30 April 2009

[19] S. Lin and D.J. Costello, "Error Control Coding: Fundamentals and Applications", Prentice-Hall, Englewood Cliffs, NJ, 1983.

[20] N. Morinaga, M. Nakagawa, and R. Kohno, "New Concepts and Technologies for Achieving Highly Reliable and High-Capacity Multimedia Wireless Communications Systems", IEEE Commun. Mag., Jan. 1997, pp. 34-40.

[21] Y. Chen, S. Kishore, and J. Li, "Wireless Diversity Through Network Coding", in Proc. WCNC, 2006, vol. 3, pp. 1681-1686.

[22] T. Wang and G. B. Giannakis, "Complex Field Network Coding for Multiuser Cooperative Communications", IEEE Journal on Selected Areas in Communications, vol. 26, pp. 561-571, April 2008.

[23] L. Xiao, T. Fuja, J. Kliewer and D. Costello, "A Network Coding Approach to Cooperative Diversity", IEEE Transactions on Information Theory, vol. 53, pp. 3714-3722, October 2007.

[24] D. Umehara, S. Denno, M. Morikura, T. Sugiyama, "Performance analysis of slotted ALOHA and network coding for single-relay multi-user wireless networks", Ad Hoc Networks, Volume 9, Issue 2, March 2011, Pages 164-179, ISSN 1570-8705, 10.1016/j.adhoc.2010.07.005.

[25] A. Munari, F. Rossetto and M. Zorzi, "Phoenix: Making Cooperation More Efficient Through Network Coding in Wireless Networks", IEEE Trans. Wireless Commun., vol. 8, no. 10, pp. 5248-5258, Oct. 2009. 
[26] K. Tan, Z. Wan, H. Zhu, J. Andrian, "CODE: Cooperative Medium Access for Multirate Wireless Ad Hoc Network", in: Proc. of the 4th Annual IEEE Communications Society Conference on Sensor, Mesh, and Ad Hoc Communications and Networks (SECON), June 2007.

[27] H.S. Mansouri, M.R. Pakravan, "Reliable and energy efficient single source broadcasting using network coding in wireless Ad-hoc networks", IEEE International Conference on Telecommunications and Malaysia International Conference on Communications (ICT-MICC), pp.81-85, 14-17 May 2007

[28] Y. Wu, P.A. Chou, Sun-Yuan Kung, "Minimum-energy multicast in mobile ad hoc networks using network coding", IEEE Transactions on Communications, vol.53, no.11, pp. 1906- 1918, Nov. 2005

[29] H. Hosseinmardi, F. Lahouti, "Online multicasting using network coding in energy constrained wireless ad hoc networks", 3rd International Symposium on Wireless Pervasive Computing (ISWPC), pp.545-549, 7-9 May 2008

[30] Sukwon Kim, T. Ho, M. Effros, "Network coding with periodic recomputation for minimum energy multicasting in mobile ad-hoc networks", 46th Annual Allerton Conference on Communication, Control, and Computing, pp.154-161, 23-26 Sept. 2008

[31] Tao Cui, Lijun Chen, T. Ho, "Energy Efficient Opportunistic Network Coding for Wireless Networks", The 27th Conference on Computer Communications. IEEE INFOCOM 2008. pp.361-365, 13-18 April 2008 
[32] L. Miao, K. Djouani, A. Kurien, G. Noel, "Network coding and competitive approach for gradient based routing in wireless sensor networks", Ad Hoc Networks, Available online 10 January 2012, ISSN 1570-8705, 10.1016/j.adhoc.2012.01.001.

[33] G. Cocco, D. Gunduz and C. Ibars, "Throughput Analysis in Asymmetric Two-Way Relay Channel with Random Access", IEEE International Conference on Communications (ICC) 2011, Kyoto, Japan, June 2011.

[34] J.-P. Ebert, S. Aier, G. Kofahl, A. Becker, B. Burns and A. Wolisz, "Measurement and Simulation of the Energy Consumption of a WLAN Interface", Technical University of Berlin,Telecommunication Networks Group, Tech. Rep. TKN-02-010, June 2002.

[35] V. Rodoplu, T.H. Meng, "Bits-per-Joule Capacity of Energy-Limited Wireless Networks", IEEE Transactions on Wireless Communications, vol.6, no.3, pp.857-865, March 2007.

[36] G. Bianchi, "Performance analysis of the IEEE 802.11 distributed coordination function", IEEE Journal on Selected Areas in Communications, vol.18, no.3, pp.535-547, Mar 2000. 